\title{
FAR INFRARED PHOTOCONDUCTIVITY OF RESIDUAL ACCEPTORS IN PURE TELLURIUM
}

\author{
K.v. Klitzing and C.R. Becker \\ Physikalisches Institut der Universität Würzburg, Germany. \\ (Received 29 March 1975; in revised form 19 March 1976 by E. Mollwo)

\begin{abstract}
The photoconductivity of both undeformed and deformed Te samples has been investigated at liquid He temperature by means of a Fourier spectrometer. Three peaks were usually found in the spectra of undeformed samples at 11,24 and $46 \mathrm{~cm}^{-1}$. These are shown to be due to three different chemical impurities. The deformed samples are characterized by additional structure at higher frequencies.
\end{abstract}

TELLURIUM is always a $p$-type semiconductor which when pure has an acceptor concentration of approximately $10^{14} \mathrm{~cm}^{-3}$. The identity and energy levels of these acceptors are usually unknown. The Hall coefficient at low temperatures is nearly constant and is therefore no help in determining the impurity binding energy. ${ }^{1}$ Infrared absorption measurements on undoped crystals show no absorption lines originating from impurities, ${ }^{2,3}$ but cyclotron resonance experiments in strong magnetic fields show impurity absorption lines identical with those found in bismuth doped crystals. ${ }^{4}$ Therefore it has been assumed that bismuth with a binding energy of about $1.4 \mathrm{meV}$ is the principal impurity in pure tellurium. On the other hand, absorption spectra of antimony doped crystals between 18 and $90 \mathrm{~cm}^{-1}$, are characterized by an absorption band at $40 \mathrm{~cm}^{-1}$. $T$ This impurity excitation energy is much higher than observed for hydrogen-like impurities originating from bismuth or antimony.

In this paper far infrared photoconductivity measurements of residual acceptor impurities in undoped tellurium are presented. This technique has been employed on several semiconductors ${ }^{6}$ and enables impurities to be detected at many orders of magnitude lower than can be detected in transmission experiments. In particular, measurements on high purity $\mathrm{Si}$ and $\mathrm{Ge}$ demonstrate that extrinsic photoconductivity provides an extremely sensitive and useful tool in determining energy levels of residual impurities. ${ }^{7}$ However in tellurium such experiments had failed because the d.c. conductivity of the most pure crystals available was so large that small variations in their resistance could not be detected. Recently the carrier concentration of our pure crystals has been reduced to about $10^{13} \mathrm{~cm}^{-3}$, and the strong surface accumulation layer, which may increase the overall conductivity of the sample by more than an order of magnitude, has been removed by a new etching process. ${ }^{8}$ Thus the d.c. conductivity was sufficiently reduced to allow the detection of a small change in the bulk resistance.

\section{EXPERIMENTAL DETAILS AND RESULTS}

The samples were cut from Czochralski grown crystals by means of a string saw employing an acid as a cutting agent. Although most of the crystals were grown in Würzburg some crystals from Wacker Chemie, München were investigated. The samples whose dimensions were typically $10 \times 2 \times 1.5 \mathrm{~mm}^{3}$ were etched in the normal manner and then rinsed with ammonium hydroxide. ${ }^{8}$ Four electrical contacts, two current leads and two potential leads, were attached and at $4.2 \mathrm{~K}$ the resistance between the two potential leads was typically between 100 and $1000 \Omega$. The measurements were carried out with a Polytec FIR 30 Fourier spectrometer while the sample was immersed in liquid helium. The photoconductive signal, the voltage drop across the potential leads of the sample under constant current conditions, was then fed into the Fourier spectrometer after being preamplified by a factor of $10^{4}-10^{5}$. In order to normalise the photoconductive signal, a background spectrum was employed with a Golay cell as a detector which has a linear energy response.

The resultant photoconductive response of one sample at 1.7 and $4.2 \mathrm{~K}$ is shown in Fig. 1. Within experimental error the higher frequency peak at $46 \mathrm{~cm}^{-1}$ is temperature independent, whereas the peak at $24 \mathrm{~cm}^{-1}$ is so strongly dependent on temperature that it has nearly disappeared at $4.2 \mathrm{~K}$. Also present is a weak peak at approximately $11 \mathrm{~cm}^{-1}$ which is much more intense at the lower temperature. The relative strengths of these maxima also depend on the current density as shown in Fig. 2. Here the photoconductive response has been normalized with respect to the total potential drop across the sample. Within experimental error the intensity of the $46 \mathrm{~cm}^{-1}$ peak is independent of current density. The $24 \mathrm{~cm}^{-1}$ peak displays a weak tendency to 


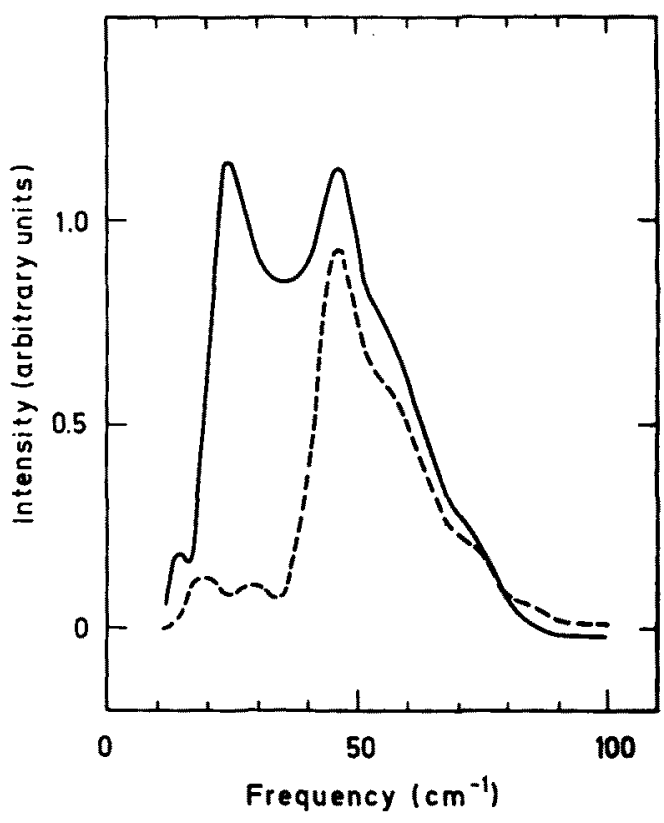

Fig. 1. Photoconductivity spectra of a Te sample at $1.7 \mathrm{~K}$ (solid curve) at $4.2 \mathrm{~K}$ (dashed curve).

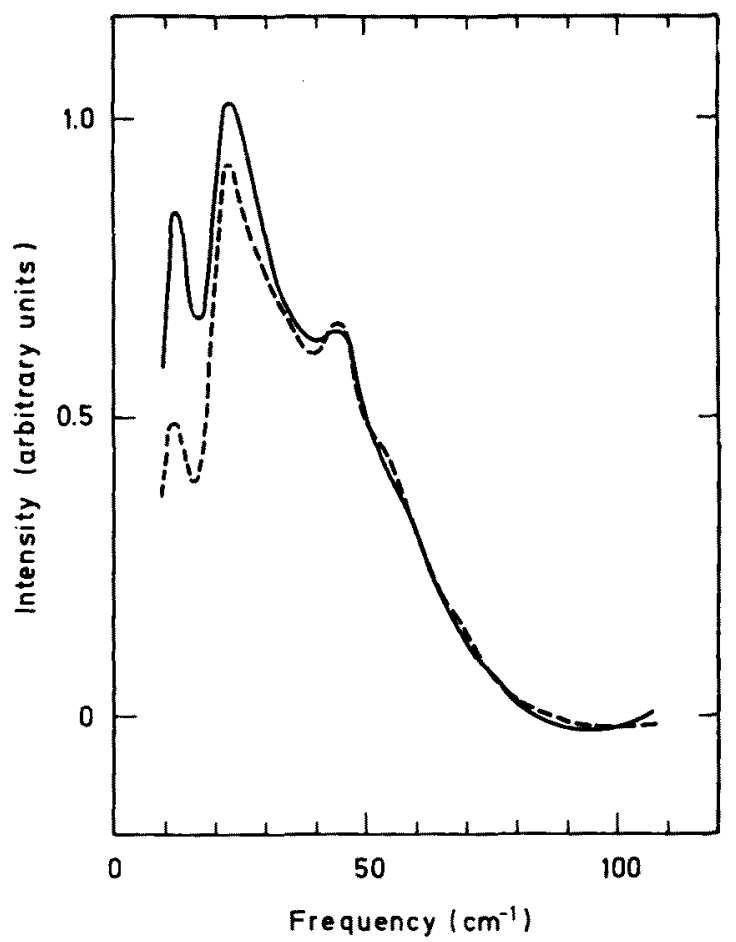

Fig. 2. Photoconductivity spectra of a Te sample at $1.7 \mathrm{~K}$ for two different currents. The photoconductive response has been normalized with respect to the total potential drop across the sample. The solid curve corresponds to $0.27 \mathrm{~mA}$ and the dashed curve to $0.75 \mathrm{~mA}$. decrease in intensity with increasing current and a strong dependence is found for the $11 \mathrm{~cm}^{-1}$ peak. These three maxima, with similar relative intensities, are present in the spectra of most of the crystals investigated. And as could be expected, the photoconductive response of samples taken from the same crystal are nearly identical.

In order to determine if these maxima are due to the presence of chemical impurities or dislocations, the photoconductive response of plastically deformed samples was investigated. The results are shown in Fig. 3 for two samples cut from the same crystal and subsequently deformed 0.9 and $3.6 \%$ by the application of uniaxial stress perpendicular to $(01 \overline{1} 0)$ or an equivalent plane at room temperature.

Three additional bands appear at 71,118 and $160 \mathrm{~cm}^{-1}$ while the original structure becomes much weaker. Care must be exercised in interpreting this new structure because of the presence of two reststrahl bands at 92 and $142 \mathrm{~cm}^{-1}$ which could lead to the appearance of three maxima. The line widths and the relative intensities of these three peaks depend on the degree of deformation; in general, one can say that the maximum at $160 \mathrm{~cm}^{-1}$ increases in intensity with increasing deformation and the two peaks at lower frequencies become broader and perhaps weaker. All deformed crystals were characterized by the three maxima in the photoconductive response mentioned above.

\section{DISCUSSION}

The general features of photoconductivity due to either isolated acceptors or donors are several sharp lines accompanied by a broad continuum at higher frequencies. ${ }^{9}$ The sharp lines which are apparently not completely resolved in this investigation, correspond to the photo-xcitation of a hole to a higher bound state followed by excitation to the valence band by the absorption of a phonon. Direct transitions to the valence band produce a broad continuum.

Theoretical calculations of the energy levels of shallow acceptors in Te have been performed by Thanh et al ${ }^{10}$ They predict a binding energy of about $1.3 \mathrm{meV}$ in agreement with cyclotron resonance ${ }^{4,11}$ and far infrared absorption ${ }^{3}$ measurements on antimony or bismuth doped crystals. This energy corresponds to our measured photoconductive maximum at $11 \mathrm{~cm}^{-1}$. Therefore we tentatively assign this peak at $11 \mathrm{~cm}^{-1}$, which was to a greater or lesser degree always present, to shallow acceptors due to the presence of antimony or bismuth.

As mentioned above, two other peaks are present in most of the undeformed samples. As can be seen in Fig. 1 the line shapes of these peaks are asymmetric with a high frequency shoulder as expected for the photoconductivity of two bound acceptors. Moreover, the fact that deformed samples are characterized by three peaks 


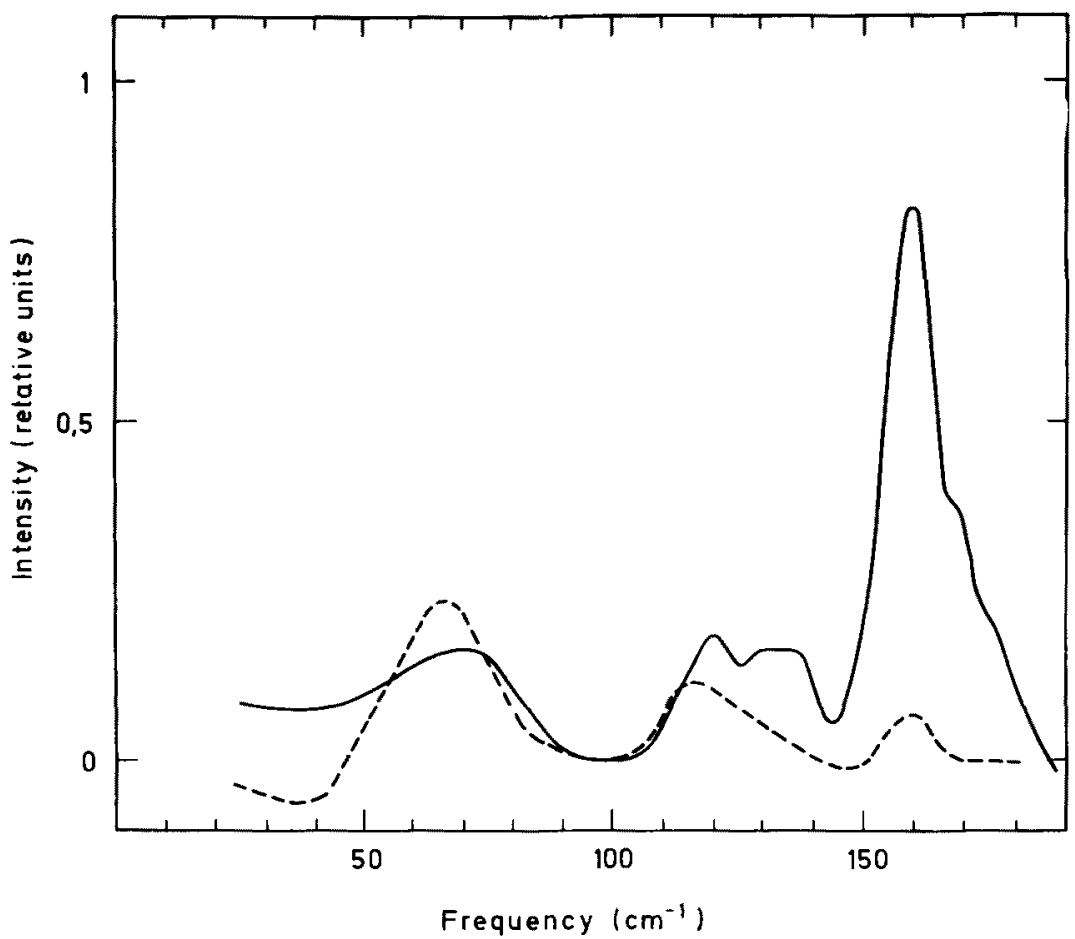

Fig. 3. Photoconductivity spectra of two deformed samples taken from the same Te crystal. The photoconductive response has been normalized with respect to the total potential drop across the samples. Dashed curve: $0.9 \%$ deformed. Solid curve: $3.6 \%$ deformed.

at higher frequencies (see Fig. 3), is an indication that the peaks at 24 and $46 \mathrm{~cm}^{-1}$ are due to chemical impurities and not dislocations.

As can be seen in Fig. 1 the peak at $24 \mathrm{~cm}^{-1}$ is so strongly temperature dependent that it has nearly disappeared at $4.2 \mathrm{~K}$, whereas the $46 \mathrm{~cm}^{-1}$ band, when a correction is made for the high frequency tail of the $24 \mathrm{~cm}^{-1}$ band, has approximately the same intensity at 1.7 and $4.2 \mathrm{~K}$. The fact that all three peaks at 11,24 and $46 \mathrm{~cm}^{-1}$ are not less intense at $1.7 \mathrm{~K}$, suggests that their corresponding excited states can not be more than about $0.5 \mathrm{meV}$ ( $3 k T$ where $T=1.7 \mathrm{~K}$ ) above the valence band, since the corresponding holes are subsequently thermally excited to the valence band at $1.7 \mathrm{~K}$. An analogous dependence on current density is shown in Fig. 2. In general, as far as the three bands are concerned, the lower their frequency the greater the influence of current density on their intensity. This is consistent with the assumption that the acceptors are ionized by the heated charge carriers. The strong temperature dependence of the $24 \mathrm{~cm}^{-1}$ band can be explained by thermal depopulation of the acceptor ground state, i.e. a removal of holes from the ground state at the higher temperature.

Recently Englert ${ }^{12}$ has shown that the temperature dependence of the Hall coefficient of deformed Te samples can be explained by one or more different types of dislocation induced acceptors with binding energies of about $15-25 \mathrm{meV}\left(120-200 \mathrm{~cm}^{-1}\right)$. He found that a $1 \%$ plastic deformation resulted in about $10^{14} \mathrm{~cm}^{-3}$ additional acceptors. The above mentioned binding energies agree well with the deformation induced photoconductive response observed in this investigation.

In summary we have shown that beside the expected hydrogen-like impurities originating from bismuth or antimony acceptors "pure" tellurium contains other impurities with excitation energies of about 24 and $46 \mathrm{~cm}^{-1}$

Acknowledgements - We wish to thank Prof. G.

Landwehr and Prof. R. Geick for helpful discussions and the Deutsche Forschungsgemeinschaft for financial support. 


\section{REFERENCES}

1. NOSKIN, V.A., FARBSHTEIN, I.I. \& SHALYT, S.S., Sov. Phys. Solid State 10, 881 (1968).

2. HARDY, D., RIGAUX, C., VIEREN, J.P. \& NGUYEN HY HAU, Phys. Status Solidi (b) 47, 643 (1971).

3. ATAKA, M., YOSHIZAKI, R. \& TANAKA, S., Solid State Commun. 13, 849 (1973).

4. COUDER, Y., HUYLIN, M. \& THOME, H., Phys. Rev. B7, 4373 (1973).

5. GROSSE, P. \& RICHTER, W., Phys. Status Solidi 41, 239 (1970).

6. BYROKA, E.M., LIFSHITS, T.M. \& SIDOROV, V.I., Sov. Phys. Semicond. 7, 671 (1973).

7. SKOLNICK, M.S., EAVES, L. \& STRADLING, R.A., Solid State Commun. 15, 1403 (1974).

8. SilbermanN, R. \& LANDWEHR, G., Proc. Int. Conf. Phys. High Magnetic Fields, p. 229. Grenoble (1974).

9. STILlMAN, G.E., WOLFE, C.M. \& DIMMOCK, J.O., Solid State Commun. 7, 921 (1969).

10. THANH, D., Solid State Commun. 9, 631 (1971).

11. YOSHIZAKI, R. \& TANAKA, S., Solid State Commun. 8, 1789 (1970).

12. ENGLERT, T., Diplomarbeit, Phys. Inst. der Univ. Würzburg (1974) (to be published).

Die photoleitung von unverformten und verformten Te Proben wurde bei he Temperaturen mit Hilfe eines Fourierspektrometers untersucht. In den Spektren der unverformten proben wurden in allgemeinen drei peaks bei 11,24 und $46 \mathrm{~cm}^{-1}$ gefunden. Es wird gezeigt, da 3 sie drei verschiedenen chemischen Verunreinigungen zuzuordnen sind. Die verformten Proben sind durch weitere Strukturen bei höneren Frequenzen charakterisiert.

Note added in proof: Measurements in a magnetic field on crystals doped with either antimony or bismuth show, in addition to a Zeeman splitting, that the peak at $11 \mathrm{~cm}^{-1}(B=0)$ is much stronger than in the undoped crystals. 